Background and Objectives ETS1 is the founder member of ETS transcription factors' family which control the transcription of various immune related genes. The aim of this study was to identify if polymorphism ETS1 Rs11221332, described in Caucasian subjects, plays any role in rheumatoid arthritis (RA) susceptibility.

Materials and Methods We genotyped this polymorphism in 136 unrelated patients with RA and 147 healthy individuals with no history of autoimmune diseases. Genotyping was performed with polymerase chain reaction-restriction fragment length polymorphism (PCR-RFLP) assay and the data were analysed by the SPSS statistical software.

Results Statistical significant difference was observed in the distribution of Rs11221332 genotypes between RA patients and controls $(p=0.041)$. Comparing Rs11221332 alleles' distribution between the studied groups the difference was higher $[p=0.039,(\mathrm{OR}=1.504$, CI:1.036-2.183)].

Conclusions The present study revealed, for first time, the positive association of a polymorphism in the sequence of ETS1 transcription factor with RA susceptibility. Further studies in other ethnic group of patients are needed to confirm the results of the present genetic association study related to ETS1, a widely used transcription factor in the regulation of various genes' expression.

\section{A7.23 THE HLA LOCUS CONTAINS NOVEL FOETAL SUSCEPTIBILITY ALLELES FOR CONGENITAL HEART BLOCK WITH SIGNIFICANT PATERNAL INFLUENCE}

doi:10.1136/annrheumdis-2013-203221.23

\begin{abstract}
1.** Sabrina Görgen, ${ }^{1, *}$ Therese Östberg, 'Stina Salomonsson, ${ }^{2}$ Bo Ding, ${ }^{3}$ Håkan Eliasson, 'Anders Mälarstig, 'Lars Alfredsson, 'Lars Klareskog, 'Anders Hamsten, ${ }^{4}$ Tomas Olsson, ${ }^{5}$ Tomas Axelsson, ${ }^{6}$ The Swedish Congenital Heart Block Study Group, ' ${ }^{1}$ redrik Gadler, ${ }^{7}$ Anders Jonzon, ${ }^{3}$ Sven-Erik Sonesson, ${ }^{4}$ Ingrid Kockum, ${ }^{1}$ Marie Wahren-Herlenius. 'Department of Medicine; ${ }^{2}$ Department of Environmental Medicine, ${ }^{3}$ Department of Women's and Children's Health; ${ }^{4}$ Department of Neuroscience, Karolinska Institutet, Stockholm; ${ }^{5}$ Department of Medical Sciences, Uppsala University; ${ }^{6}$ www.combinesweden.se; 'Department of Women's and Children's Health, Uppsala University, Uppsala, Sweden
\end{abstract}

*These authors contributed equally

Objective To identify foetal susceptibility genes in Ro/SSA autoantibody-mediated congenital heart block on chromosome six. Methods Single nucleotide polymorphism (SNP) genotyping of individuals included in the Swedish Congenital Heart Block (CHB) study population was performed. Low resolution HLA-A, -Cw and -DRB1 allele typing was carried out in 86 families of the study population comprising 339 individuals (86 Ro/SSA autoantibody positive mothers, 71 fathers, $87 \mathrm{CHB}$ index cases and 95 unaffected siblings). Results A case-control comparison between index cases and population-based out of study controls $(n=1710)$ revealed an association of CHB with fifteen SNPs in the 6p21.3 MHC locus at a chromosome-wide significance of $\mathrm{p}<2.59 \times 10^{-6}$ (OR 2.21-3.12). In a family-based analysis between SNP markers as well as distinct MHC class I and II alleles with $\mathrm{CHB}$ we observed associations to HLA-DRB $1 * 04$ and HLA-Cw*05 variants that were significantly more often transmitted to affected individuals $(p<0.03$ and $p<0.05$, respectively), and HLA-DRB1*13 and HLA-Cw*06 variants which were significantly less often transmitted to affected children $(p<0.05$ and $p<0.04)$. We further observed a significant association of increased paternal, but not maternal, HLA-DRB1*04 transmissions to the affected offspring $(p<0.02)$.

Conclusions Our study identifies HLA-DRB1*04 and HLA-Cw*05 as novel foetal HLA-allele variants that confer susceptibility to develop $\mathrm{CHB}$ in response to exposure to Ro/SSA autoantibodies, while DRB1*13 and $\mathrm{Cw}^{*} 06$ emerged as protective alleles. For the first time, we also demonstrate paternal contribution to foetal susceptibility to $\mathrm{CHB}$.

\section{A7.24 THE PENTANUCLEOTIDE INSERTION IN HSPA1B GENE IS ASSOCIATED WITH IDIOPATHIC INFLAMMATORY MYOPATHY}

doi:10.1136/annrheumdis-2013-203221.24

T Svitalkova, M Remakova, M Faustova, L Plestilova, H Mann, J Vencovsky, P Novota. Institute of Rheumatology, Prague, Czech Republic

Background and Objectives The HSPA1B gene is one of the three HSP70 genes located within the Major Histocompatibility Complex (MHC) on chromosome 6p21. The HSP70 molecules in their intracellular form have antiapoptotic function and are responsible for stabilisation of protein structure; in their extracellular form, they act as mediators of immune response. The extracellular HSPs are part of the innate and adaptive immune response and are involved in the process of antigen presentation. The aim of our study was to find out if an association between polymorphisms of MHC located HSP70 genes and subgroups of idiopathic inflammatory myopathy exists.

Materials and Methods We have analysed 177 patients suffering from idiopathic inflammatory myopathy (82 patients with dermatomyositis - DM, 71 patients with polymyositis - PM, 22 patients with cancer associated myositis, 2 patients with inclusion body myositis) and 59 healthy controls. In total, six genetic polymorphisms located within the three HSP70 genes were analysed by direct genomic DNA sequencing. The statistical analysis was done using Fisher's exact test with calculated $\mathrm{p}<0.05$ considered as statistically significant.

Results and Conclusions The frequency of the "INS" allele of the pentanucleotide insertion polymorphism in HSPA1B (Rs9281590) was increased in patients suffering from myositis (43.79\%) in comparison with controls $(32.20 \%$; $<<0.05)$. The Odds Ratio calculated for this polymorphism was 1.64 (CI95\% 1.056; 2.545). Its increased frequency was predominantly found in DM patients $(\mathrm{p}<0.05)$; the allele distribution in PM patients did not significantly differ from controls. Presence of INS allele was strongly related to the well described HLA associated risk, the HLA-DRB1*03 allele $(p<0.001)$, found mostly in PM patients. INS allele is independent on other myositis associated HLA allele, HLA-DRB1*16, found increased in the population of our DM patients. Other polymorphisms analysed in this study did not show any relation to the myositis. Our findings support the hypothesis, that DM and PM have partially different genetic background.

Acknowledgement This work is supported by Internal Grant Agency of Ministry of Health of the Czech Republic NT/13699.

\section{A7.25 GENE-GENE INTERACTIONS IN INTERFERON PATHWAY GENE POLYMORPHISMS IN EUROPEAN AND AMERICAN SCLERODERMA COHORTS}

doi:10.1136/annrheumdis-2013-203221.25

'Pravitt R Gourh, ${ }^{2}$ Filemon K Tan, ${ }^{3}$ Blanca Rueda, ${ }^{2}$ Frank C Arnett, ${ }^{2}$ Shervin Assassi, ${ }^{2}$ John D Reveille, ${ }^{4}$ Timothy RD Radstake, ${ }^{2}$ Sandeep K Agarwal, ${ }^{5}$ Javier Martin; ${ }^{2}$ Maureen D Mayes. 'National Institutes of Health, Bethesda, MD; ${ }^{2}$ University of Texas Health Science Center at Houston, Houston, TX; ${ }^{3}$ Facultad de Ciencias de la Salud. Universidad de Granada, Granada, Spain; ${ }^{4}$ University Medical Centre Utrecht, Utrecht, The Netherlands; ${ }^{5}$ Instituto de Parasitologia y Biomedicina Lopez-Neyra (CSIC), Granada, Spain

Background/Purpose Type-I interferon (IFN), a central mediator of innate immunity, has been shown to be the hallmark peripheral blood gene expression pattern in lupus (SLE) and a similar type-I IFN signature has been noted in systemic sclerosis (SSc). Interferon regulatory factor 5 and 7(IRF5/IRF7) and tyrosine kinase 2(TYK2) are important genes involved in this signalling cascade. The purpose of this work was to investigate the association and interaction of IRF5, IRF7, TYK2 polymorphisms with SSc. 